\title{
Slowly evolving Kaposi's sarcoma in a patient newly diagnosed with HIV infection
}

\author{
Eugenia-Andreea Marcu ${ }^{1,2}$, Amalia Romanescu², Florentina Dumitrescu ${ }^{1,2}$ \\ ${ }^{1}$ University of Medicine and Pharmacy, Craiova, Romania \\ 2"Victor Babes" Clinical Hospital of Infectious Diseases and Pneumoftiziology, Craiova, \\ Romania
}

\begin{abstract}
Introduction. Kaposi's sarcoma (KS) is a multicentric vascular neoplasia typically occurring in the context of immunodeficiency. Although the incidence of HIV-related KS has dramatically decreased and the prognosis improved due to the combined antiretroviral therapy (cART), KS remains an important cause of morbidity and mortality among people living with HIV.

Case presentation. We describe the case of a-34-year-old patient from an urban area, who is admitted in the Dermatology Clinic from Emergency Clinical County Hospital Craiova reporting a 3-years history of painful infiltrated plaques on the left hand, in the groin area and on the left lower limb. Among the biological investigations ELISA test-HIV1,2 is performed, with a positive result so the patient is transferred to the HIVI AIDS Department - „Victor Babes“ Clinical Hospital of Infectious Diseases and Pneumoftiziology Craiova. The patient underwent treatment with: antiretrovirals, antibiotics, antifungals, anticoagulants, vasodilators and symptomatic drugs. During hospitalization, the patient also developed violaceous lesions in the oral cavity, on the right foot and on the left ear lobe. Skin biopsy was performed and the histopathological examination confirmed the clinical suspicion of KS. The oncological treatment was initiated after about 2 months and the evolution of the patient has been favorable, with remission of mucocutaneous lesions more than $50 \%$.

Conclusions. Persistent skin lesions in a young patient can be an indicator marker for AIDS and the delayed HIV diagnosis may influence the prognosis.
\end{abstract}

Keywords: Kaposi's sarcoma, HIV, antiretroviral therapy

\section{INTRODUCTION}

Kaposi's sarcoma (KS) is a multicentric vascular neoplasia typically occurring in the context of immunodeficiency. It was first described in 1872 by the physician Moritz Kaposi (1).

In the U.S. and Europe classic KS was considerred, for most of the 20th century, as an indolent neoplasm. Its aggressive evolution was first noted among homosexuals, as part of acquired immunodeficiency syndrome (AIDS) (2).

$\mathrm{KS}$ is frequently evident as multiple cutaneous and mucosal lesions, which are infiltrated and violaceous and tend to evolve (3). There are four types of
KS now widely recognised: classic KS, which has an indolent course and occurs predominantly in Mediterranean and Middle Eastern men; epidemic KS, in patients infected with the human immunodeficiency virus (HIV) with severe immunosuppression, who have a CD4 cell count below 500 cells $/ \mathrm{mm}^{3}$ and it may be the first sign of HIV infection; african KS (endemic), whose incidence has increased tens of times since the onset of the AIDS epidemic.; iatrogenic KS (transplant-related KS) $(4,5)$.

In 2018, according to the data of the World Health Organization, globally there were 41,799 people newly diagnosed with KS and 19,902 deaths due to KS (6). 
Although the incidence of HIV-related KS has dramatically decreased and the prognosis improved due to the combined antiretroviral therapy (cART), KS remains an important cause of morbidity and mortality among people living with $\operatorname{HIV}(7,8)$.

There is clinical evidence indicating that $\mathrm{KS}$ lesions may disappear or regress when cART is introduced $(9,10)$. Complete remission of KS occurs in $20 \%$ to $80 \%$ of patients and is more common among cART-naive patients, with early-stage disease, who have compliance to cART (11). In patients with rapidly progressive disease, cART should be combined with chemotherapy (12). However, in some HIV-positive patients, KS may recrudesce early following the introduction of cART as an immune reconstitution inflammatory syndrome (IRIS) (13).

Prior to the introduction of cART, various therapeutic agents were used in the treatment of KS such as interferon-alpha, thalidomide, vincristine, bleomycin. However, the introduction of liposomal anthracyclines in the early 1990s led to a significant progress in KS therapy, becoming the gold standard. Their role was reinforced by the evidence in three randomized studies, which showed better response rates and a lower toxicity compared to previously used chemotherapeutic agents. In the advanced stages of KS, cART in combination with liposomal anthracyclines improves response rate to $70 \%$ (14).

British HIV Association Guidelines suggests that patients with early-stage of KS should be treated with cART alone, reserving the addition of chemotherapy in advanced stages, based on its effectiveness in HIVpositive patients with visceral KS. The effectiveness of this stage-stratified approach was strengthened by a study of 469 patients, confirming that this approach resulted in high survival rates in patients with advanced stage disease and it reduced the exposure to chemotherapy in patients with early-stages of KS $(15,16)$.

Despite the progress of systemic management of $\mathrm{KS}$, up to $15 \%$ of patients develop progressive disease, even they have sustained immuno-virological control of HIV infection, with undetectable HIV viral load (VL-HIV) and a CD4 cell count greater than 500 cells $/ \mathrm{mm}^{3}$ (17).

\section{CASE PRESENTATION}

We describe the case of a-34-year-old patient from an urban area, who is admitted in the Dermatol- ogy Clinic from Emergency Clinical County Hospital Craiova reporting a 3-years history of painful infiltrated plaques on the left hand, in the groin area and on the left lower limb. The patient also complains of dysuria, pollakiuria, symptoms that occurred 5 days ago.

Among the biological investigations ELISA testHIV1,2 is performed, with a positive result so the patient is transferred to the HIV/AIDS Department „Victor Babes” Clinical Hospital of Infectious Diseases and Pneumoftiziology Craiova.

The objective examination at the time of admission (October 2019) reveals: severe general condition, violaceous plaque on the left foot with edema and ascending lymphangitis to the groin area, violaceous plaque on the left hand, penile edema, lymphadenopathy in the groin area, cardiopulmonary balanced, oxygen saturation $=97 \%$, heart rate $=83$ beats per minute, blood pressure $=124 / 76 \mathrm{mmHg}$, adherent white plaques on the tongue, abdominal tenderness on palpation, no signs of meningeal irritation.

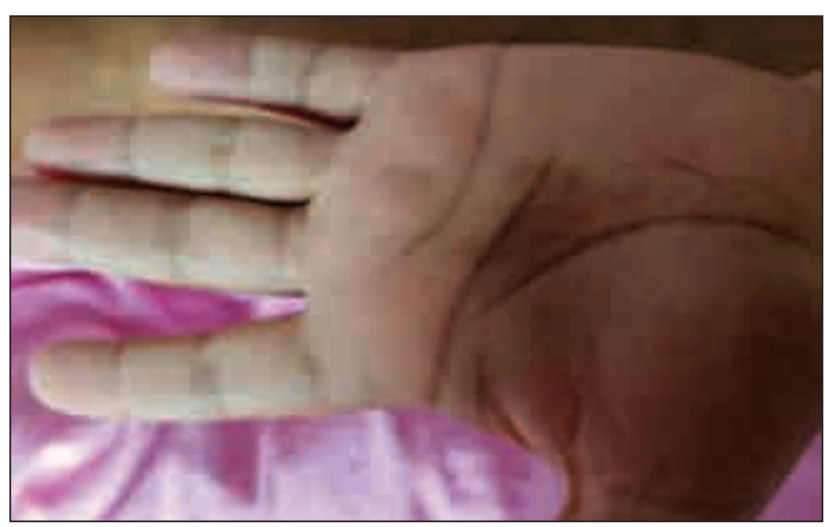

FIGURE 1. Palmar skin lesions

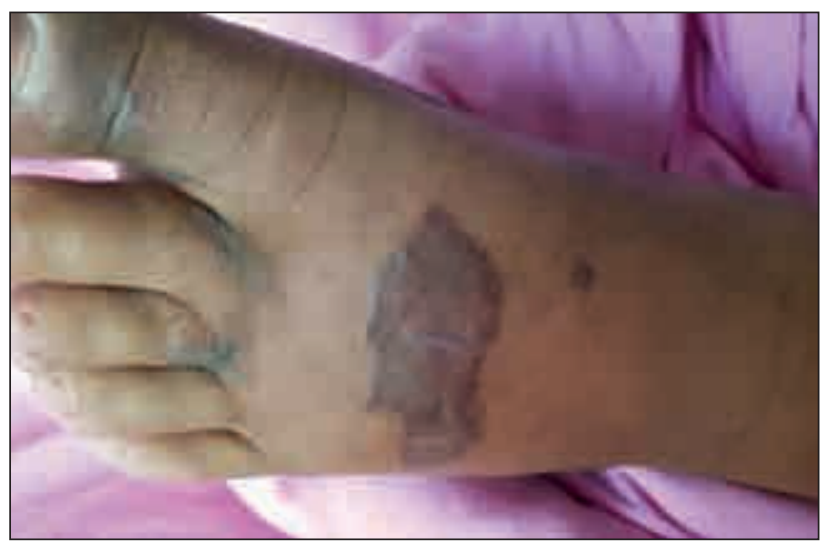

FIGURE 2. Skin lesions on the dorsum of the foot

Laboratory tests show an inflammatory syndrome $(\mathrm{ESR}=82 \mathrm{~mm} / 1 \mathrm{~h}, 110 \mathrm{~mm} / 2 \mathrm{~h}, \mathrm{C}$-reactive protein $=$ 


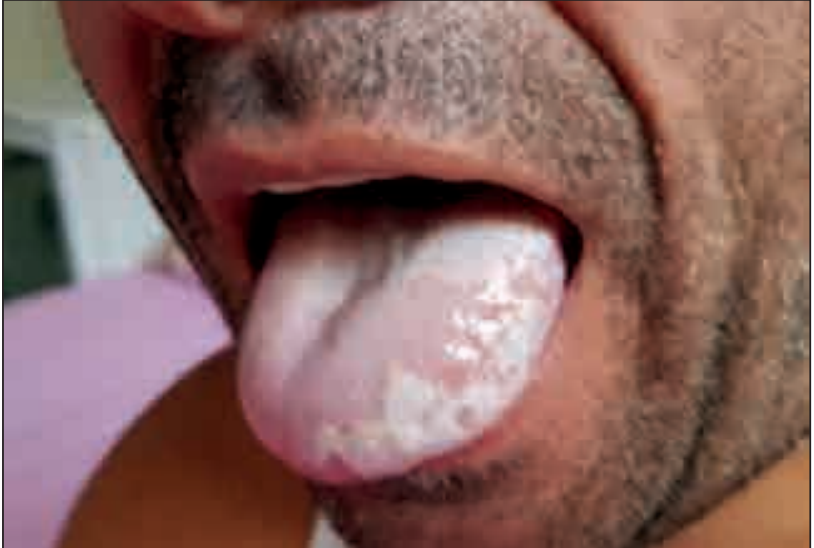

FIGURE 3. Oral lesions

$12 \mathrm{mg} / \mathrm{l}$ ), fungal culture from lingual exudate Candida albicans (antifungal susceptibility: Fluconazole, Amphotericin B), sputum examination BK negative, urethral discharge - fungal culture $=$ Candida albicans, urine culture - Escherichia coli (antibiotic susceptibility: Cefoxitin, Meropenem, Ciprofloxacin), Ag HBs-absent, HCV Atc - absent, Toxoplasma gondii, cytomegalovirus, Epstein-Barr antibodies IgG positive, VL-HIV $=247,000$ copies/ $\mathrm{ml}, \mathrm{CD} 4$ count $=244$ cells $/ \mathrm{mm}^{3}$.

MRI scan reveals infiltration of subcutaneous tissue in the left lower limb.

Surgical consultation: edema and bruising caused by vascular disorders. Dermatological consultation is recommended for skin biopsy with histopathological examination.

Dermatological consultation: KS under observation. Oral hairy leukoplakia. Oral candidiasis. Skin biopsy is performed.

Stage diagnosis: HIV infection-newly diagnosed case. KS under observation. Oral candidiasis. Oral hairy leukoplakia. Urinary tract infection with Escherichia coli.

Evolution and treatment: During hospitalization, the patient also developed violaceous lesions in the oral cavity, on the right foot and on the left ear lobe and he is transferred to the Intensive Care Unit for monitoring.

The patient underwent treatment with: antibiotics (Clarithromycin $500 \mathrm{mg} / 12$ hours - 17 days, Meronem $1 \mathrm{~g} / 8$ hours -10 days, Linezolid 1 vial/12 hours -7 days), antifungals (Fluconazole $400 \mathrm{mg} / 24$ hours 10 days), anticoagulants, vasodilators and symptomatic drugs.

HIV drug resistance was performed using genotyping testing. It has been identified a low-level resistance to nucleoside reverse transcriptase inhibi- tors: didanosine (ddI), zidovudine (AZT). The mutations E44D, T215E were associated with drug resistance.

Antiretroviral therapy was initiated after 10 days of hospitalization with Emtricitabine/Tenofovir + Dolutegravir.

Two weeks after the biopsy, the result of the histopathological examination is received, which highlights a tissue fragment whose epithelium has acanthosis and underlying lymphocytic infiltrate; microscopic structure of KS.

The CT scan of the chest, abdomen and pelvis with contrast agent don't show focal parenchymal lesions and adenomegaly.

The patient is discharged after 3 weeks, with improved general condition, afebrile, smooth scar (after skin biopsy), cardiopulmonary balanced, with the recommendation to continue cART and monitoring and therapeutic care through Oncology Department.

Diagnoses upon discharge: HIV infection category C2. Mucocutaneous KS. Oral candidiasis. Oral hairy leukoplakia. Urinary tract infection with Escherichia coli.

After about 2 months, first-line chemotherapy with Paclitaxel and Carboplatin is introduced, the patient being undergoing to 10 cures of treatment.

One year after initiation of cART, the patient has undetectable VL-HIV, CD4 cell count $=329$ cells $/ \mathrm{mm}^{3}$. The evolution has been favorable, with remission of mucocutaneous lesions more than 50\%.

\section{DISCUSSIONS}

Optimal management of HIV-related KS requires an early diagnosis of KS and an effective therapeutic approach to prevent complications and disease progression, as well as to improve patients' prognosis.

In a cohort study conducted in UK between 1996 and 2008, a group of 254 HIV-positive patients diagnosed with KS was analysed. $96 \%$ of them were male and the mean age at KS diagnosis was 39 years. At KS diagnosis, $19 \%$ of patients were on cART, only $7 \%$ of patients had an undetectable plasma VL-HIV and $122(48 \%)$ had CD4 cell count $<150$ cells $/ \mathrm{mm}^{3}$. 163 patients were treated with cART alone and only 37 required chemotherapy. The overall 5-year survival was $89 \%$ (18).

Another study was performed in Nigeria and included 96 HIV-positive patients, of whom 48 presented HIV-related KS. The average age of the 
patients was $36 \pm 8$ years and $41.7 \%$ were male. The median CD4 lymphocytes count was 96 cells $/ \mathrm{mm}^{3}$ [2:306] compared to 126 cells $/ \mathrm{mm}^{3}$ [9:743] among HIV-positive patients without KS. $77.1 \%$ of patients presented generalized lesions. Patients with HIVrelated KS had higher mortality (19).

$\mathrm{KS}$ can develop in HIV-positive patients who are naive to cART, with normal CD4 cell count. Initiating cART in patients with higher CD4 cell count may reduce morbidity and mortality among this patients (20).

In France, Lomé, it was performed a study between January 2005 - October 2011 on 157 patients treated in the dermatology departments for KS. HIV serology was positive for $103(89.6 \%)$ of the 115 patients tested. 79 patients were known to be HIV-positive, while KS was the first manifestation of the HIV infection for 24 patients. The mean age of the 103 patients was $36.7 \pm 14.9$ years and the sexratio $(\mathrm{M} / \mathrm{F})$ was 1.1 . The main locations of the lesions were the lower limbs (76 patients), mucosa (53 patients), trunk (38 patients) and upper limbs (17 patients). The average CD4 cell count was $226 \pm 168$ cells $/ \mathrm{mm}^{3}$. The most commonly used cART was stavudine/lamivudine/nevirapine (70 patients). Besides the cART, patients also received chemotherapy with vinblastine (17 patients), bleomycin (5 patients) and doxorubicin (one patient). For financial reasons, 80 other patients did not receive chemotherapy. At 5 months, the rate of complete remission was $21.1 \%$, partial remission $21.1 \%$ and failure $57.8 \%$. Side effects were dominated by hematologic and nervous complications (21).

\section{REFERENCES}

1. Ferla L, Pinzone MR, Nunnari G, et al. Kaposi's sarcoma in HIV-positive patients: the state of art in the HAART-era. Eur Rev Med Pharmacol Sci. September 2013;17(17):2354-65.

2. Simard EP, Engels EA. Cancer as a cause of death among people with AIDS in the United States. Clin Inf Dis. 2010;51(8):957-62.

3. Schwartz RA,Micali G, Nasca MR, et al. Kaposi sarcoma: a continuing conundrum. J Am Acad Dermatol. 2008;59(2):179-206.

4. Cook-Mozaffari P, Newton R, Beral V, et al. The geographical distribution of Kaposi's sarcoma and of lymphomas in Africa before the AIDS epidemic. Br J Cancer. 1998;78(11):1521-8.

5. Reddy D, Mitsuyasu R. HIV-associated multicentric Castleman disease. Current Opin Oncol. 2011;23(5):475-81.

6. WHO. - Globocan 2018. Availble at: https://gco.iarc.fr/today/data/ factsheets/populations/900-world-fact-sheets.pdf.

7. Gallafent JH, Buskin SE, de Turk PB, et al. Profile of patients with Kaposi's sarcoma in the era of highly active antiretroviral therapy. J Clin Oncol. 2005;23(6):1253-60.

8. Holkova B, Takeshita K, Cheng DM, et al. Effect of highly active antiretroviral therapy on survival in patients with AIDS-associated pulmonary Kaposi's sarcoma treated with chemotherapy. J Clin Oncol. 2001;19(18):3848-51.
In our patient's case, macrolide therapy was instituted because we've taken into account bacillary angiomatosis (later refuted by the histopathological result). The extension of lesions after starting cARTcould indicate the onset of IRIS, the patient presenting risk factors for the development of IRIS (young age, male gender, low CD4 cell count at cART initiation).

Unlike the other forms of KS, HIV-related KS occurs in young patients and the lesions have a rapid progression, with visceral involvement in $50 \%$ of cases.

Our patient's skin lesions did not have the classic appearance of KS, which led to a delayed diagnosis, this being favored by the long time between lesions onset and hospital presentation.

\section{CONCLUSIONS}

Persistent skin lesions in a young patient can be an indicator marker for AIDS and the delayed HIV diagnosis may influence the prognosis. Screening of these patients is mandatory to prevent the disease progression, even if the skin lesions are localized.

Strategies and measures have been developed to optimize the process of diagnosis and treatment of KS associated with HIV infection, such as the early introduction of antiretroviral therapy, interdisciplinary collaboration to reduce the time for the diagnosis of KS and updating the old therapeutic protocols to include more effective treatments, with lower toxicity.

9. Gill J, Bourboulia D, Wilkinson J, et al. Prospective study of the effects of antiretroviral therapy on Kaposi sarcoma - associated herpesvirus infection in patients with and without Kaposi sarcoma. J Acquir Immune Defic Syndr. 2002;31(4):384-90.

10. Paparizos VA, Kyriakis KP, Papastamopoulos V, et al. Response of AIDS-Associated Kaposi Sarcoma to Highly Active Antiretroviral Therapy Alone. J Acquir Immune Defic Syndr. 2002;30(2):257-8.

11. Mosam A, Shaik F, Uldrick TS, et al. A randomized controlled trial of highly active antiretroviral therapy versus highly active antiretroviral therapy and chemotherapy in therapy-naive patients with HIV-associated Kaposi sarcoma in South Africa. J Acquir Immune Defic Syndr. 2012;60(2):150-7.

12. Martín-Carbonero L, Barrios A, Saballs $P$, et al. Pegylated liposomal doxorubicin plus highly active antiretroviral therapy versus highly active antiretroviral therapy alone in HIV patients with Kaposi's sarcoma. AIDS. 2004;18(12):1737-40.

13. Feller L, Anagnostopoulos C, Wood NH, et al. Human immunodeficiency virus-associated Kaposi sarcoma as an immune reconstitution inflammatory syndrome: a literature review and case report. J Periodontol. 2008;79(2):362-8. 
14. Stewart S, Jablonowski H, Goebel FD, et al. Randomized comparative trial of pegylated liposomal doxorubicin versus bleomycin and vincristine in the treatment of AIDS-related Kaposi's sarcoma. International Pegylated Liposomal Doxorubicin Study Group. J Clin Oncol. 1998;16(2):683-91.

15. Bower M, Palfreeman A, Alfa-Wali M, et al. British HIV Association guidelines for HIV-associated malignancies 2014. HIV Med. 2014; 15(2):1-92.

16. Bower M, Dalla Pria A, Coyle C, et al. Prospective stage-stratified approach to AIDS-related Kaposi's sarcoma. J Clin Oncol. 2014; 32(5):409-14.

17. Stebbing J, Powles T, Bower M. AIDS-associated Kaposi's sarcoma associated with a low viral load and a high CD4 cell count. AIDS. 2008;22(4):551-2.
18. Bower M, Weir J, Francis N, et al. The effect of HAART in 254 consecutive patients with AIDS-related Kaposi's sarcoma. AIDS. August 2009;23(13):1701-6.

19. Agaba PA, Sule HM, Ojoh RO, et al. Presentation and survival of patients with AIDS-related Kaposi's sarcoma in Jos, Nigeria. Int $\mathrm{J}$ STD AIDS. June 2009;20(6):410-3.

20. Nasti G, Martellotta F, Berretta M et al. Impact of highly active antiretroviral therapy on the presenting features and outcome of patients with acquired immunodeficiency syndrome-related Kaposi sarcoma. Cancer. 2003;98:2440-6.

21. Saka B, Mouhari-Toure A, Wateba IM, et al. AIDS related Kaposi sarcoma: 103 cases in dermatology in Lomé (Togo). Med Sante Trop. January 2013;23(1):109-11. 